Acta Crystallographica Section A

Foundations of Crystallography

ISSN 0108-7673

Received 1 April 2005

Accepted 8 June 2005

\section{Polarization anisotropy of $\mathrm{X}$-ray atomic factors and 'forbidden' resonant reflections}

\author{
Vladimir E. Dmitrienko, ${ }^{a *}$ Kohtaro Ishida, ${ }^{\mathrm{b}}$ Armin Kirfel ${ }^{\mathrm{c}}$ and \\ Elena N. Ovchinnikovad
}

\begin{abstract}
${ }^{\mathbf{a}} \mathrm{A}$. V. Shubnikov Institute of Crystallography, 119333 Moscow, Russia, ${ }^{\mathbf{b}}$ Faculty of Science and Technology, Tokyo University of Science, Noda, Chiba 278-8510, Japan, ' Universität Bonn, Poppelsdorfer Schloss, D-53115 Bonn, Germany, and Physical Department of Moscow State University, 119899 Moscow, Russia. Correspondence e-mail: dmitrien@ns.crys.ras.ru
\end{abstract}

\begin{abstract}
Symmetry and physical aspects of 'forbidden' reflections excited by a local polarization anisotropy of the X-ray susceptibility are surveyed. Such reflections are observed near absorption edges where the anisotropy is caused by distortions of the atomic electronic states owing to interaction with neighbouring atoms. As a consequence, they allow for extracting nontrivial information about the resonant atom's local environment and their physical conditions. The unusual polarization properties of the considered reflections are helpful to distinguish them from other types of 'forbidden' reflections. When such reflections are excited, it is, for example, possible to determine not only the intrinsic anisotropy of an atomic form factor but also additional anisotropy induced by thermal motion, point defects and/or incommensurate modulations. Even the local 'chirality' of atoms in centrosymmetric crystals is accessible. Unsolved key problems and possible future developments are addressed.
\end{abstract}

(C) 2005 International Union of Crystallography Printed in Great Britain - all rights reserved contrasts. A much more complicated situation arises, however, when the radiation energy approaches an absorption edge. Then, prior to the production of photoelectrons, core electrons can be excited into vacant states above the Fermi level, which depend on chemical bonding and are distorted by the local environment. As a consequence, $f^{\prime}$ and $f^{\prime \prime}$ of the 'edge atom' turn into tensors, which leads to a complex atomic scattering factor (ASF), to structure-factor tensors in general and hence to special polarization-dependent diffraction.

In 1980, Templeton \& Templeton noticed that an anisotropy of resonant scattering, i.e. an anisotropic X-ray susceptibility, can give rise to the excitation of so-called 'forbidden' reflections otherwise systematically absent owing to the presence of symmetry elements with translation components, i.e. screw axes and glide planes. The physics of this phenomenon is fairly clear: the incident waves 'see' symmetry-related but differently oriented ASF tensors giving rise to non-vanishing structure-factor tensors and violations of extinction rules owing to screw rotations and glide reflections. This effect may be considered an unwanted complication that can be circumvented by simply avoiding the say $50 \mathrm{eV}$ wide region of resonance energies but, as immediately recognized by Templeton \& Templeton (1982), 'where there is a complication there is also the opportunity of sharper, more penetrating methods for extracting information from diffraction experiments'. And indeed, while anisotropic resonant scattering principally affects all Bragg reflections, the 'forbidden' ones have attracted most attention in the past two decades, mainly because they are exclusively signals from the partial structure 
of the edge atom(s) and thus most sensitive element and sitespecific probes of the atomic local environments.

Following a general theoretical treatment of the 'forbidden' reflections by Dmitrienko $(1983,1984)$ and the first experimental verification, namely the observation of the excitement of 'forbidden' $00 l(l=2 n+1)$ reflections in cubic $\mathrm{NaBrO}_{3}$ (Templeton \& Templeton, 1985a, 1986), similar effects have been found and studied in various other crystals like $\mathrm{Cu}_{2} \mathrm{O}$ (Eichhorn et al., 1988), $\mathrm{TiO}_{2}, \mathrm{MnF}_{2}$ (Kirfel et al., 1991), $\mathrm{LiHSeO}_{3} \quad$ (Kirfel \& Petcov, 1992), $\mathrm{Ba}\left(\mathrm{BrO}_{3}\right)_{2} \cdot \mathrm{H}_{2} \mathrm{O}$ (Templeton \& Templeton, 1992), $\mathrm{Fe}_{3} \mathrm{O}_{4}$ (Kirfel et al., 1995a; Hagiwara et al., 1999; García et al., 2000; García, Subías et al., 2001), $\mathrm{FeS}_{2}$ (Nagano et al., 1996; Templeton \& Templeton, 1997; Kokubun, Nagano et al., 1998), $\mathrm{HoFe}_{2}$ (Collins et al., 2001); for further details see below. Such experiments are time consuming because once a resonance is detected the transition has to be proved and characterized by means of energy scans in steps of about $1 \mathrm{eV}$ and numerous measurements at different polarizations and different $\psi$ settings in order to reveal the azimuthal variation of the 'forbidden' reflection's intensity in the often very disturbing presence of generally much larger multiple scattering effects. In a theoretical treatment within the dipole-dipole approximation, explicit expressions were developed for calculating the intensity and polarization properties of 'forbidden' reflections and results have been compiled in tabular form for space groups up to tetragonal symmetry (Kirfel \& Morgenroth, 1993; Morgenroth et al., 1994a).

Most of the experimental studies involved dipole-dipole $(d d)$ transitions, but also higher-order transitions like the much weaker pure quadrupole-quadrupole $(q q)$ and/or mixed dipole-quadrupole $(d q)$ transitions have been observed, e.g. in hematite, $\alpha-\mathrm{Fe}_{2} \mathrm{O}_{3}$ (Finkelstein et al., 1992; Watanabe et al., 2001; Kokubun, Watanabe et al., 2005), and in magnetite, $\mathrm{Fe}_{3} \mathrm{O}_{4}$ (Kirfel et al., 1995a; Hagiwara et al., 1999; García et al., 2000; Kanazawa et al., 2002; Subías, García, Proietti et al., 2004), where the polarization and spectral analysis of the scattered radiation revealed site-specific $d q$ transitions for the pre-edge peak. The latter type was also assumed to cause the 'forbidden' $h k 0(h+k=4 n+2)$ reflections in the diamond structure of germanium (Templeton, 1994; Templeton \& Templeton, 1994) but later it was found (Kokubun et al., 2001; Kirfel et al., 2002b) that a thermal-motion-induced $d d$ contribution is the reason for this class of reflections, at least at ambient and elevated temperatures (see $\$ 8$ below).

This paper presents a survey of the observations of the pure crystalline 'forbidden' reflections with emphasis on the experimental features; their theoretical treatment has been reviewed before by Belyakov \& Dmitrienko (1989), Carra \& Thole (1994) and Dmitrienko \& Ovchinnikova (2003). Problems related to 'forbidden' reflections induced by magnetic and orbital ordering can be found elsewhere [see, for instance, Lovesey \& Collins (1996) and Ishihara \& Maekawa (2002)]. In fact, calculations show that structural distortions (probably of the Jahn-Teller type) are the main reasons for 'forbidden' reflections near the Mn $K$ edge in crystals with orbital ordering like $\mathrm{LaMnO}_{3}$ (Elfimov et al., 1999; Benfatto et al., 1999; Takahashi et al., 1999) as experimentally confirmed by García, Sánchez et al. (2001) and Herrero-Martín et al. (2004). Orbital ordering can be directly probed by soft X-ray resonant scattering at the $\mathrm{Mn} L_{\mathrm{II}}$ and $L_{\mathrm{III}}$ edges (Wilkins et al., 2003); however, such activities are beyond the scope of our present paper.

\section{2. 'Forbidden' reflections}

Conditions limiting possible reflections (extinction rules) were calculated many years ago for all crystallographic space groups and are listed in International Tables for Crystallography (1996), Vol. A. They follow from the phase differences between the atomic scattering amplitudes of symmetryequivalent atoms positioned at different points in the unit cell, and those differences depend on the crystal symmetry and the occupied atomic sites. Systematic absences of certain types of reflections (called forbidden reflections or extinctions) allow for discriminating between the space groups (though not always unambiguously).

More generally, however, the sets of allowed and forbidden reflections depend not only on the crystal symmetry and structure but also on details of the physical interaction between the incoming and scattered radiation and the atoms in the unit cell. In fact, the conditions listed in International Tables apply only to point scalar scatterers placed at the atomic positions or to atoms with spherically symmetric electron-density distributions. If one takes, however, into account distortions of the electronic states by neighbouring atoms, then the structure amplitudes of the 'forbidden' reflections can deviate from zero and the extinction conditions should be modified. The best known example is the 222-type reflection in the diamond structure (see, for instance, Dawson, 1975), which is excited because the atomic electron-density distribution is distorted by chemical bonding to the neighbouring atoms or by anharmonic thermal motion. Other examples are supplied by pure magnetic reflections observed in magnetic crystals by neutron (Izyumov et al., 1991), X-ray (Gibbs et al., 1985; Hannon et al., 1988; Lovesey \& Collins, 1996) and Mössbauer diffraction (Belyakov, 1975) or by crystals with orbital ordering (Ishihara \& Maekawa, 2002; Wilkins et al., 2003).

For the sake of clarity, one has therefore to distinguish between different types of the reflection conditions listed in International Tables. The general conditions apply to atoms in general positions in the unit cell and may be of two types: (i) conditions related to pure translation symmetry, i.e. lattice centring, which are obviously strict in any case regardless of the type of radiation; and (ii) conditions related to the presence of glide planes and screw axes. Additional (special) conditions (iii) apply to atoms in special positions. These latter conditions are easily violated, for example by non-spherical atomic electron-density distributions. Indeed, the nonspherical parts of the atomic electron density can be considered as small 'pseudo-atoms' in general positions. The 222type reflections in the diamond structure are just of this type (iii). 
Therefore, a non-trivial situation arises for the reflections violating conditions (ii) related to the presence of glide planes and/or screw axes. These general conditions cannot be violated by a distortion of the electron density compatible with the crystal space group because the electron density is invariant under the glide-plane and screw-axis symmetry operations. In particular, they cannot be violated even if the electron density is distorted by chemical bonding and/or by thermal motion because all such distortions (on average) retain the space group. The conditions (ii) can, however, be violated if, in a more general approach, the interaction of $\mathrm{X}$-rays with an atom is described by the tensor of susceptibility rather than by a scalar quantity or, in other words, by a tensorial atomic scattering factor (ASF). The reason for a violation is that, in general, the ASF tensor changes the orientation of its principal axes under the glide-plane and screw-axis symmetry operations of the space group so that the ASF tensors of different, though symmetry-related, atoms in the unit cell cannot compensate each other. The scalar description is in principle only valid if one considers the electrons as free, which is a good approximation for most practical purposes as long as the X-ray energy is sufficiently far from absorption edges. Near an absorption edge, this approximation fails because the tensorial ASF can become noticeably anisotropic and, as will be discussed below, 'forbidden' reflections can be excited as a direct consequence of this anisotropy. Before this, however, the physics of the polarization anisotropy will be addressed.

\section{Physical reasons for the polarization anisotropy}

Suppose as usual that the different atoms of a structure scatter independently so that the X-ray diffraction is described by a tensorial structure factor $\hat{F}$ :

$$
F_{j k}\left(\omega, \mathbf{k}, \mathbf{k}^{\prime}\right)=\sum_{s} f_{j k}^{s}\left(\omega, \mathbf{k}, \mathbf{k}^{\prime}\right) \exp \left(i \mathbf{H} \mathbf{r}^{s}\right),
$$

where $\mathbf{r}^{s}$ is the position of the sth atom in the unit cell, the atomic factor $f_{j k}^{s}\left(\omega, \mathbf{k}, \mathbf{k}^{\prime}\right)$ is a second-rank tensor depending, in general, on the radiation frequency $\omega$ and the wavevectors $\mathbf{k}$ and $\mathbf{k}^{\prime}$ of the incident and diffracted waves, and $\mathbf{H}=\mathbf{k}^{\prime}-\mathbf{k}$ is the scattering vector. The amplitude of diffraction, from an initial polarization e to a diffracted polarization $\mathbf{e}^{\prime}$, is proportional to $e_{j}^{\prime *} F_{j k} e_{k}$, and the intensity of the diffracted radiation is proportional to $\left|e_{j}^{\prime *} F_{j k} e_{k}\right|^{2} / \mu$, where $\mu(\omega)$ is the linear absorption coefficient (here, $\mu$ is assumed isotropic and the diffraction experiment is in Bragg geometry using a sample plate of practically infinite thickness).

The atomic factors in equation (1) may be divided into two parts: the main isotropic (scalar) part is due to the potential or Thomson scattering from the electron density, whereas the resonant contribution (the so-called anomalous correction) includes the binding effects. This latter part $f_{j k}$ (we consider only the resonant term and omit index $s$ ) can be written as

$$
f_{j k}\left(\omega, \mathbf{k}, \mathbf{k}^{\prime}\right)=-\frac{m}{\hbar \omega} \sum_{a, b} p_{a} \omega_{b a}^{3} \frac{\left\langle a\left|O_{j}^{*}\right| b\right\rangle\left\langle b\left|O_{k}\right| a\right\rangle}{\omega-\omega_{b a}-i \Gamma / 2 \hbar} ;
$$

see, for instance, Blume (1994) for details. In this expression, $|a\rangle$ describes the initial and final electronic states with energy $E_{a}, p_{a}$ is a probability of finding the atom in the initial $|a\rangle$ state, $|b\rangle$ describes an intermediate electronic state with energy $E_{b}, \omega_{b a}=\left(E_{b}-E_{a}\right) / \hbar$; the vectors $\mathbf{O}$ and $\mathbf{O}^{\prime}$ include two terms, dipole and quadrupole: $O_{j}=D_{j}+i Q_{j m} k_{m} / 2, O_{j}^{\prime}=$ $D_{j}+i Q_{j m} k_{m}^{\prime} / 2$, where $\mathbf{D}_{j}=\sum_{i} \mathbf{r}^{i}$ and $Q_{j m}=\sum_{i} r_{j}^{i} r_{m}^{i}$, and the summation extends over all electrons in the atom (see also Brouder, 1990). Correspondingly, there are three types of terms in $f_{j k}\left(\omega, \mathbf{k}, \mathbf{k}^{\prime}\right)$ : dipole-dipole $(d d)$, dipole-quadrupole $(d q)$ and quadrupole-quadrupole $(q q)$. The initial and final states coincide because only elastic coherent scattering is considered.

For X-ray energies, the initial states $|a\rangle$ correspond to electrons at inner levels that are practically undistorted by crystalline fields. In contrast, the intermediate electronic states, a few eV above the Fermi level, can be distorted by the local environment because the virtual photoelectrons strongly interact (scatter) with neighbouring atoms. As a consequence, the ASF tensor becomes anisotropic with a symmetry corresponding to the site symmetry of the resonant atom. This anisotropy is most pronounced in the direct vicinity of an absorption edge (i.e. in the XANES region) but it may also occur in the EXAFS region, whereas it is negligible far from the edges. Therefore, non-resonant atoms can be neglected in calculations of 'forbidden' reflection structure factors.

Explicit calculations with equation (2) are rather complicated because of multiple scattering of the virtual photoelectrons by neighbouring atoms. Several codes have been developed for this type of calculation in the XANES region (Natoli et al., 1990; Vedrinskii et al., 1992; Ankudinov et al., 1998; Benfatto \& Felici, 2001; Joly, 2001; Taillefumier et al., 2002), which, in principle, allow for a quantitative description of the ASF tensor (see examples below). However, in practice, this description must be improved with respect to the experimental energy profiles. The other approach, namely the symmetry-based phenomenological description (considered in the next section) can be used universally for predicting and evaluating 'forbidden' reflections, but it cannot provide quantitative fits to the resonance curves.

\section{Symmetry restrictions on atomic and structure factors}

In general, the structure-factor tensor of any reflection is a second-rank tensor with nine independent complex elements. This number reduces upon taking into account the internal tensor symmetry: for instance, in the $d d$ approximation, the tensor is symmetric for non-magnetic crystals so that the number of its independent components is six. Further reduction of the number of independent components follows when the crystal space group and/or the local site symmetry of the atomic positions (external symmetries) are also taken into account. Since the spatially periodic susceptibility tensor is intrinsically invariant against the symmetry operations of the crystal space group, it has been shown (Dmitrienko, 1983) for the $d d$ approximation that the structure factors of the glide- 
plane and screw-axis 'forbidden' reflections can contain at most two independent components, in many cases even less as, for instance, in crystals with cubic space groups where the structure factors become additionally constrained (Dmitrienko, 1984).

The most convenient and traditional way is to start with the tensorial $\operatorname{ASF} f_{j k}$ and to expand it as a function of $\mathbf{k}$ and $\mathbf{k}^{\prime}$,

$$
f_{j m}\left(\omega, \mathbf{k}, \mathbf{k}^{\prime}\right)=f_{j m}^{d d}+i f_{j m n}^{d q s}\left(k_{n}^{\prime}-k_{n}\right)+i f_{j m n}^{d q a}\left(k_{n}^{\prime}+k_{n}\right)+\ldots,
$$

followed by the $q q$ term. The tensorial coefficients $\hat{f}$ (denoted as $f$ tensors) are of different rank and possess different internal symmetries: for example, the $d d$ tensor $f_{j m}^{d d}$ is always symmetric $\left(f_{j m}^{d d}=f_{m j}^{d d}\right)$ if time-reversal symmetry is assumed, whereas the $d q$ are general terms that may be represented by sums of symmetric $\left(f_{j m n}^{d q s}=f_{m j n}^{d q s}\right)$ and antisymmetric $\left(f_{j m n}^{d q a}=-f_{m j n}^{d q a}\right)$ contributions.

Normally, the $d d$ term dominates the other terms of the expansion making them difficult to detect. The $d q$ and $q q$ terms are usually much smaller because the sizes of the inner shells are smaller than the wavelength. Sometimes, however, the $d d$ term does not contribute at all to the structure factor of a 'forbidden' reflection and then the higher-order terms become decisive (see $\$ 7$ ).

Since the anisotropy of the $f$ tensors in equation (3) is caused by the atomic environments, the $f$ tensors should conform with the site symmetries of the actual atomic positions, which are not necessarily equilibrium positions. Their forms for second, third and fourth ranks can be found in textbooks (Sirotin \& Shaskolskaya, 1975; Sirotine \& Chaskolskaia, 1982, 1984; Nye, 1985; International Tables for Crystallography, 2003, Vol. D) for all crystallographic point groups.

Atoms in equivalent sites obviously have equivalent $f$ tensors but the orientations of the principal tensor axes can be different owing to different orientations of the atoms' environments. Therefore, one has to define the $f$ tensor of one of the 'edge atoms' considered, according to the site symmetry of its position, denote it as $\hat{f}^{1}$, then find the $f$ tensors of all other equivalent atoms in the unit cell by application of the spacegroup-symmetry operations and finally calculate the tensorial structure factor $\widehat{F}$. For example, in the $d d$ approximation, one has

$$
\widehat{F}(\omega, \mathbf{H})=\sum_{s} \widehat{R}\left(g^{s}\right) \hat{f}^{1}(\omega) \widehat{R}\left(g^{s}\right)^{-1} \exp \left(i \mathbf{H r}^{s}\right) .
$$

The summation is over all the equivalent resonant atoms in the unit cell and $\widehat{R}\left(g^{s}\right)$ is the rotational or mirror part of the symmetry operation $g^{s}$ that relates the position of atom $s$ to the position of atom 1 . For non-equivalent positions, the $f$ tensors are not related at all.

Let us illustrate this procedure with a simple example: the $d d$ approximation for pyrite crystallizing in space group $P a \overline{3}$. In the pyrite unit cell, the four Fe atoms lie on the threefold axes, i.e. in $4 a$ positions with point symmetry $\overline{3}$. Thus, the $\mathrm{Fe}$ scattering-factor tensors must be uniaxial. Let atom 1 be the atom at the origin of the unit cell, $\mathbf{r}^{1}=(0,0,0)$, on the 3 axis parallel to [111]. Its atomic factor is given by the uniaxial tensor

$$
\hat{f}^{1}=\left(\begin{array}{lll}
f_{x x} & f_{x y} & f_{x y} \\
f_{x y} & f_{x x} & f_{x y} \\
f_{x y} & f_{x y} & f_{x x}
\end{array}\right),
$$

where $f_{x x}$ is the isotropic part of the atomic factor tensor whereas $f_{x y}$ describes the anisotropy of $\hat{f}^{1}$. With $f_{\|}$and $f_{\perp}$ denoting the eigenvalues of $\hat{f}^{1}$ in the directions parallel and perpendicular to the threefold axis, respectively, $f_{x y}=$ $\left(f_{\|}-f_{\perp}\right) / 3, \quad f_{x x}=\left(f_{\|}+2 f_{\perp}\right) / 3$. Obviously, $\hat{f}^{1}$ is invariant against threefold rotation about [111], i.e. against cyclic permutations of $x, y, z$.

The tensorial atomic factors of the other three Fe atoms, 2, 3 and 4 , are related to $\hat{f}^{1}$ by twofold rotations about the $x, y$ and $z$ axes correspondingly:

$$
\begin{aligned}
\hat{f}^{2} & =\left(\begin{array}{ccc}
f_{x x} & -f_{x y} & -f_{x y} \\
-f_{x y} & f_{x x} & f_{x y} \\
-f_{x y} & f_{x y} & f_{x x}
\end{array}\right), \\
\hat{f}^{3} & =\left(\begin{array}{ccc}
f_{x x} & -f_{x y} & f_{x y} \\
-f_{x y} & f_{x x} & -f_{x y} \\
f_{x y} & -f_{x y} & f_{x x}
\end{array}\right), \\
\hat{f}^{4} & =\left(\begin{array}{ccc}
f_{x x} & f_{x y} & -f_{x y} \\
f_{x y} & f_{x x} & -f_{x y} \\
-f_{x y} & -f_{x y} & f_{x x}
\end{array}\right) .
\end{aligned}
$$

Then, using equation (1) yields the structure-factor tensors of the $00 l(l=2 n+1)$ screw-axis 'forbidden' reflections as well as of the $0 k l(k=2 n+1)$ glide-plane 'forbidden' reflections:

$$
\begin{gathered}
\widehat{F}(00 l)=\frac{4}{3}\left(f_{\|}-f_{\perp}\right)\left(\begin{array}{lll}
0 & 0 & 0 \\
0 & 0 & 1 \\
0 & 1 & 0
\end{array}\right), \\
\widehat{F}(0 k l)=\frac{4}{3}\left(f_{\|}-f_{\perp}\right)\left(\begin{array}{lll}
0 & 0 & 1 \\
0 & 0 & 0 \\
1 & 0 & 0
\end{array}\right) .
\end{gathered}
$$

As to be expected, the scalar parts (diagonal elements) cancel and thus do not contribute to the 'forbidden' reflections.

Finally, let us consider the polarization and azimuthal dependence of the diffraction (in the kinematical approximation, which is usually sufficient for the 'forbidden' reflections). Although in the $d d$ approximation there is no azimuthal dependence of $F_{j k}$, the intensity usually exhibits a harmonic $\psi$ dependence because both the initial and the diffracted polarizations, $\mathbf{e}$ and $\mathbf{e}^{\prime}$, depend on the azimuthal crystal setting $\psi$. For the cases $d q$ and $q q$, there may be additional azimuthal terms owing to inherent $k$ dependencies of the respective ASF tensors themselves. The polarization properties of the reflections are also determined by the tensor form of the structure factor $F_{j k}$. For the 'forbidden' reflections, they are rather unusual: for instance, in the case of a $2_{1}$ screw axis, an incident $\boldsymbol{\sigma}$-polarized radiation produces a totally $\boldsymbol{\pi}$ polarized diffracted wave (in the $d d$ approximation). Notice 
that in some cases, as for all 'forbidden' reflections in pyrite, the forms of the tensors do not change with the X-ray energy [equations (9) and (10)] so that the azimuthal polarization properties are also energy independent.

Detailed discussions of the polarization and azimuthal properties have been given by Dmitrienko (1983), Kirfel et al. (1991) and Kirfel \& Morgenroth (1993). The polarization dependence of $\mu$ is usually neglected but if important it can be included as discussed by Kirfel \& Petcov (1992).

\section{Glide-plane and screw-axis 'forbidden' reflections in different crystals}

The first 'forbidden' reflections of this type were observed in an $\mathrm{NaBrO}_{3}$ crystal (Templeton \& Templeton, 1985a, 1986). Similar reflections were known for light diffraction in chiral liquid crystals, where they were called 'selective reflections' (Belyakov et al., 1979), and from Mössbauer diffraction (Belyakov, 1975) but in both cases the anisotropy is not as small as for X-rays. The space group of $\mathrm{NaBrO}_{3}$ is $P 2_{1} 3$, the resonant $\mathrm{Br}$ atoms are in four equivalent positions on the threefold axes, $\mathbf{r}^{1}=x, x, x$ etc. Calculations similar to those in the above pyrite example yield, for the $00 l(l=2 n+1)$ reflections,

$$
|\boldsymbol{\pi} \widehat{\mathbf{F}} \boldsymbol{\sigma}|^{2}|\boldsymbol{\sigma} \widehat{\mathbf{F}} \boldsymbol{\pi}|^{2}=\frac{8}{9}\left|f_{\|}-f_{\perp}\right|^{2} \cos ^{2} \theta(1-\cos 4 \pi l x \cos 2 \psi),
$$

where $\theta$ is the Bragg angle. In this case, the polarization properties are the same for all reflections and all azimuthal angles $\left(\boldsymbol{\sigma} \rightarrow \boldsymbol{\pi}^{\prime}\right.$ and $\left.\boldsymbol{\pi} \rightarrow \boldsymbol{\sigma}\right)$ and were not explicitly recorded. The azimuthal dependencies of three screw-axis 'forbidden' reflections are shown in Fig. 1. The absolute scale in Fig. 1 is based on the allowed 006 reflection. Notice that in a cubic crystal in the absence of linear dichroism (at least in the $d d$ approximation) these observations allow for the experimental determination of $\left|f_{\|}-f_{\perp}\right|$. Another interesting feature is the dependence of the intensity on the atom's position ( $x$ coordinate), a circumstance that can be used for partial structure analysis as is discussed in $\S 6$.

Since the first observations of 'forbidden' reflections in $\mathrm{NaBrO}_{3}$, numerous synchrotron-radiation studies have been performed, mostly on crystal structures with cubic, tetragonal or hexagonal symmetry and containing 'edge atoms' above $Z=22$. They served mainly to detect anisotropic resonant scattering, to show the possibility of exciting 'forbidden' reflections and to prove the validity of the optical model yielding the azimuthal dependencies of the scattered radiation in terms of both intensity and polarization. Except for cubic crystals, the search for suitable candidates may be preceded by checking for encouraging polarization-dependent absorption or fluorescence as described by Templeton \& Templeton for $\mathrm{VO} \cdot 2\left(\mathrm{C}_{5} \mathrm{H}_{7} \mathrm{O}_{2}\right)(1980), \mathrm{RbUO}_{2}\left(\mathrm{NO}_{3}\right)_{3}(1982), \mathrm{C}_{6} \mathrm{H}_{12} \mathrm{~N}_{2} \mathrm{O}_{4} \mathrm{Se}$ (1988), $\mathrm{LiIO}_{3}(1989 a, b)$, and also by other authors for $\mathrm{LiNbO}_{3}$ (Kirfel \& Petcov, 1988; Petcov et al., 1988; Petcov, 1989; Petcov et al., 1990) or minerals (Lippmann et al., 1998) and synthetic compounds (Lippmann et al., 1992, 1994, 1995).
Examples of more detailed studies of 'forbidden' reflections are given below.

Cuprite, $\mathrm{Cu}_{2} \mathrm{O}$, crystallizes in space group $P n \overline{3} m$ with $\mathrm{Cu}$ on the threefold axes being linearly coordinated by two $\mathrm{O}$ atoms. Thus, the $\mathrm{Cu}$ environment is highly anisotropic giving rise to well observable resonant 'forbidden' reflections $0 \mathrm{kl}$, $k+l=2 n+1$. The axial reflections $00 l(l=2 n+1)$ in particular have been extensively studied (Kirfel et al., 1991; Eichhorn et al. 1988; Kirfel, Petcov et al., 1988; Kirfel \& Eichhorn, 1988, 1989; Hock, Kirfel \& Lippmann, 1995; Hock, Lippmann \& Kirfel, 1995) including the refinement of the $f^{\prime}, f^{\prime \prime}$ tensor elements (Morgenroth et al., 1993) and proving the observability of anisotropic resonant scattering by powder diffraction measurements (Kirfel, Eichhorn \& Wroblewski, 1988; Lippmann \& Kirfel, 1991). Recent quantitative studies (Ninomiya et al., 2001) revealed a very large anisotropy of $\left|f_{\|}-f_{\perp}\right| \approx 5.7$ e at most.
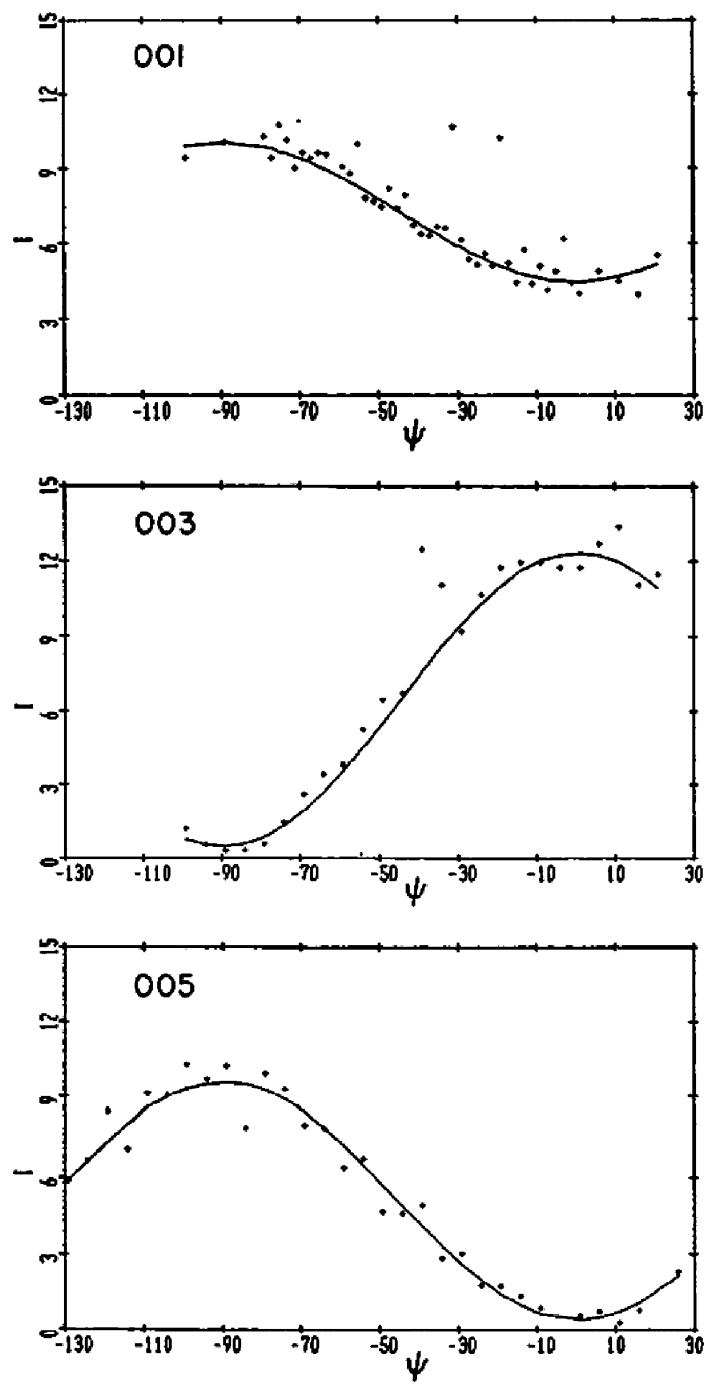

Figure 1

Observed (points) and calculated (lines) squares of structure factors for 001, 003 and 005 plotted as a function of azimuthal angle, at a photon energy $8 \mathrm{eV}$ below the $\mathrm{Br} K$ white-line peak. A few high points, and another too far off scale to plot, indicate coincidence with Renninger reflections; this is Fig. 1 by Templeton \& Templeton (1986). 
Magnetite, $\mathrm{Fe}_{3} \mathrm{O}_{4}$, space group $F d \overline{3} m$, crystallizes in the inverse spinel structure with the $\mathrm{Fe}$ atoms occupying both octahedral and tetrahedral sites. This offers the possibility of observing 'forbidden' reflections excited by a $d q$ transition which is only allowed on the tetrahedral site whereas the octahedral site allows, on the contrary, only for $d d$ and $q q$ transitions (Kirfel et al., 1995a; Fischer, Krane \& Kirfel, 1996). By polarization analysis of the reflection 208 measured at the energies of the two distinct Fe $K$-edge resonance lines, it could be shown that one originates from the octahedral, the other from the tetrahedral site. This point has also been confirmed by Kanazawa et al. (2002) who studied both magnetite and franklinite $\mathrm{ZnFe}_{2} \mathrm{O}_{4}$ and have shown that the pre-edge peak disappears and zinc gives no 'forbidden' reflections in $\mathrm{ZnFe}_{2} \mathrm{O}_{4}$ where the Fe atom occupies only the octahedral site. Similar results have been found by García \& Subías (2004) for $\mathrm{MnFe}_{2} \mathrm{O}_{4}$, whereas, for $\mathrm{CoFe}_{2} \mathrm{O}_{4}$ with $\mathrm{Co}$ atoms occupying the octahedral sites, the pre-edge $\mathrm{Fe}$-atom peak is very pronounced. Low-temperature studies of magnetite (Hagiwara et al., 1999; García et al., 2000; García, Subías et al., 2001; Renevier et al., 2001; García \& Subías, 2004; Subías, García, Blasco et al., 2004) have claimed absence of charge ordering and enforced re-examination of the classical picture of the Verwey transition although this problem is still rather controversial (Nazarenko et al., 2005). In another crystal of the same space group, $\mathrm{HoFe}_{2}$, where $\mathrm{Ho}$ and $\mathrm{Fe}$ atoms respectively occupy the tetrahedral and octahedral sites, the 'forbidden' reflections were found only at the $\mathrm{Fe} K$ edge (Collins et al., 2001).

Also, rutile types $\mathrm{TiO}_{2}$ and $\mathrm{MnF}_{2}$, space group $\mathrm{P}_{2} / \mathrm{mnm}$, exhibit well observable effects (Kirfel et al., 1989, 1991; Kirfel \& Petcov, 1989) owing to a quite small deviation of the cation coordination polyhedron from full octahedral symmetry. A reinvestigation of rutile and studies of the anisotropic resonant scattering in the related $\mathrm{TiO}_{2}$ anatase and brookite structures (Sawai et al., 2003) demonstrate how different resonant 'forbidden' reflections can be in the same chemical compound, at both pre-edge and edge energies (Fig. 2), although the absorption spectra (XANES) of the crystals are similar. While rutile and brookite show $d d$-type resonances at

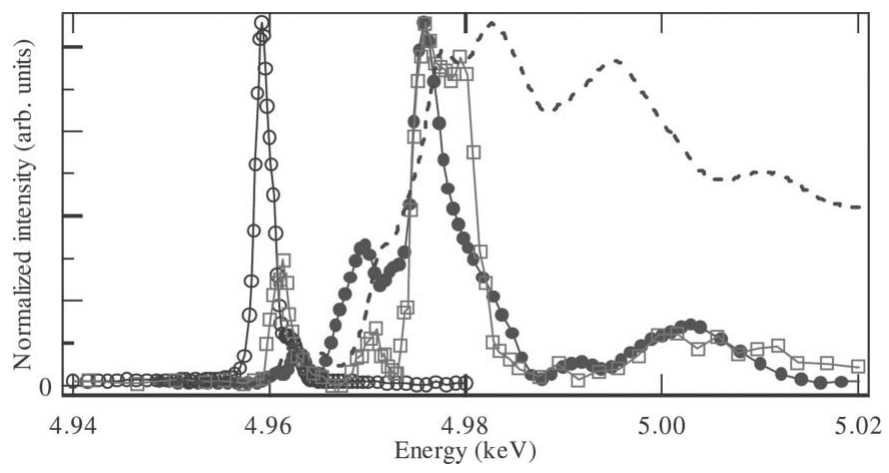

Figure 2

The energy dependence of 'forbidden' reflections from different $\mathrm{TiO}_{2}$ structures near the Ti absorption edge: rutile 100 (black circles); anatase 002 (open circles); brookite 300 (squares); the absorption edge for rutile is shown by the dashed line (Kokubun \& Ishida, 2005). the Ti $K$ edge, anatase does not because the space-group symmetry $P 4_{1} /$ amd precludes a $d d$ term.

Pyrite crystals, $\mathrm{FeS}_{2}$, space group $\mathrm{Pa} \overline{3}$, were studied in several papers. Nagano et al. (1996) analysed the polarization properties of reflections 001 and 011 from plates cut correspondingly. Later, Kokubun, Nagano et al. (1998) carefully studied the reflection spectra 001, 003, 011 and their azimuthal dependence. The $d d$ contribution was shown to be dominant above the edge whereas the pre-edge resonance was revealed as $q q$. Very similar 'forbidden' reflection spectra were found recently for pyrite-like $\mathrm{CoS}_{2}$ and $\mathrm{NiS}_{2}$ (Kokubun \& Ishida, 2005). Templeton \& Templeton (1997) have studied the azimuthal profiles of 'forbidden' and weak allowed reflections from a small irregular sample $(0.15 \times 0.19 \times 0.20 \mathrm{~mm})$. The incident polarization was supposed to be completely $\boldsymbol{\sigma}$. The glide-plane 'forbidden' reflections 520 and 530 were measured for $7120.4 \mathrm{eV}$, and the absolute value $\left|f_{\|}-f_{\perp}\right|$ was determined from theoretical fits and comparison with the non-forbidden reflection 332. Measurements for the weak non-forbidden reflection 432, where the anisotropic contribution is of the order of the isotropic one, were carried out at several energies, and both the real and imaginary parts of the anisotropic ASF (and hence its phase) could be determined this way. Another method to measure the phase, namely via interference with Renninger reflections (Kokubun et al., 2004), is discussed at the end of this section.

Careful polarization analysis of diffracted beams was also performed for the 005, 007 and 0,0,10 reflections from a hexagonal ferrite, $\left(\mathrm{Ba}_{1-x} \mathrm{Sr}_{x}\right)_{2} \mathrm{Zn}_{2} \mathrm{Fe}_{12} \mathrm{O}_{22}$ (Tsuji et al., 1996). From this analysis and by comparison of the peak intensity with that of the allowed 006 reflection, the tensorial structure factor of the 0,0,10 reflection was obtained. Magnetic satellites were also studied in this paper.

A remarkably strong $d d$-type resonance occurs in $\operatorname{Sr}\left(\mathrm{NO}_{3}\right)_{2}$, space group $P a \overline{3}$ (Fischer \& Kirfel, 1997). With Sr in $(0,0,0)$, site symmetry $\overline{3}$, this effect can be rationalized in terms of the $\mathrm{O}$ atoms coordinating $\mathrm{Sr}$ atoms in the form of a cubo-octahedron distorted along the [111] direction. Not yet fully understood among the hexagonal symmetry examples is the case of $\mathrm{LiNbO}_{3}$, for which the excitement of 'forbidden' $h \bar{h} 0 l$ reflections $(l=2 n+1)$ has been observed using diffraction in Laue geometry from a platelet cut parallel to [0001] (Petcov, 1989). The fact that these results could not be reproduced by measurements in Bragg geometry on a (0001) face is still a challenge for future work.

Studies of lower-symmetry crystals are rarer, but need to be mentioned. An example is $\mathrm{Ba}\left(\mathrm{BrO}_{3}\right)_{2} \cdot \mathrm{H}_{2} \mathrm{O}$, space group $I 2 / c$ (Templeton \& Templeton, 1992). The azimuthal profiles for several glide-plane 'forbidden' reflections were measured near the $\mathrm{Br} K$ edge and the value of local anisotropy was determined from fitting theoretical curves and scaling to the allowed 006 reflection. Further examples are orthorhombic $\mathrm{LiHSeO}_{3}$ (Kirfel et al., 1990; Lippmann et al., 1992; Kirfel \& Lippmann, 1992; Kirfel \& Petcov, 1991, 1992), $\mathrm{KH}_{3}\left(\mathrm{SeO}_{3}\right)_{2}$ (Fischer \& Kirfel, 1998), $\mathrm{CuSeO}_{3} \cdot 2 \mathrm{H}_{2} \mathrm{O}$ (Kirfel \& Lippmann, 1994), $\mathrm{Na}_{2}\left[\mathrm{Fe}(\mathrm{CN})_{5}(\mathrm{NO})\right] \cdot 2 \mathrm{H}_{2} \mathrm{O}$ (Kirfel et al., 1995b; Lippmann et al., 1995), $\mathrm{PbZrO}_{3}$ (Toda et al., 1998) and monoclinic 
Tutton salts $A_{2} B\left(\mathrm{SO}_{4}\right)_{2} \cdot 6 \mathrm{H}_{2} \mathrm{O}$ with $A=\mathrm{K}, \mathrm{NH}_{4}$ and $B=\mathrm{Fe}$, $\mathrm{Co}, \mathrm{Ni}, \mathrm{Cu}$ (Fischer et al., 1996a,b; Fischer \& Kirfel, 1997). Most of these studies were undertaken with the aim to find candidates with 'edge atoms' in lower site-symmetry positions and to explore the possibility to use 'forbidden' reflections and their azimuthal intensity variations for partial structure determination, which is addressed in the next section.

Here, it should be noted that, owing to experimental difficulties and the underlying objective of most studies, both in verifying the developed model and in finding new candidates with reasonably large effects, the majority of the experiments has been carried out at ambient conditions. Only in recent years has it turned out that temperature is also an important parameter.

Low-temperature measurements have been reported for cuprite (Kirfel \& Krane, 1999) and magnetite (Hagiwara et al., 1999; García, Subías et al., 2001; Kirfel et al., 2002a) but, as shown in $\S 8$, studies at elevated temperatures may also make an essential contribution.

As mentioned before, ATS is most pronounced in the XANES region. It can, however, also be observed at higher energies in the EXAFS region where its structural interpretation is more obvious (García et al., 2000; Collins et al., 2003; Subías, García, Proietti et al., 2004).

Recently, it was also shown that the resonant reflections can be helpful in understanding the charge ordering in the mixedvalence transition-metal compound $\mathrm{NaV}_{2} \mathrm{O}_{5}$, phase transition from Pmmn symmetry to $A 112$ (Joly et al., 2003). Even fine details of the charge ordering could be revealed by a quanti-
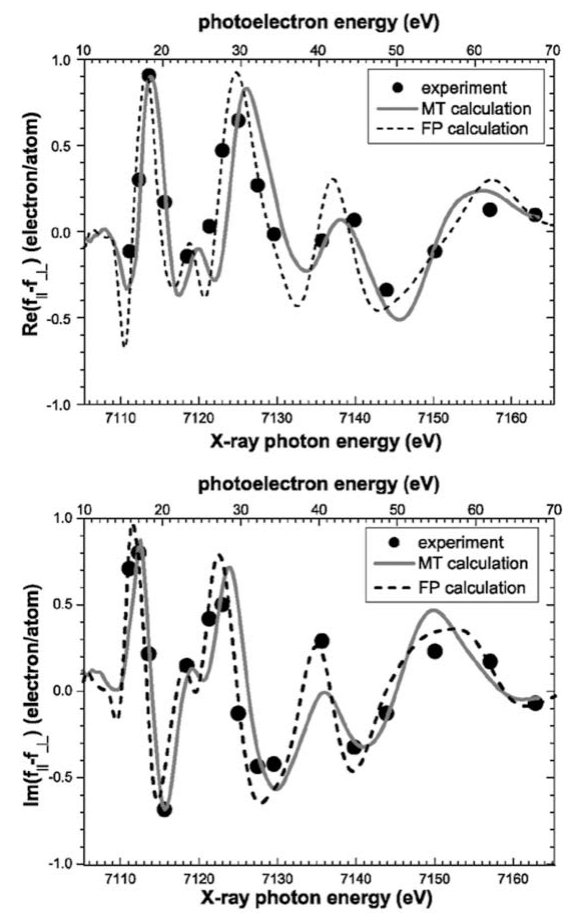

Figure 3

Experimental values of the local anisotropy of the atomic factor (on absolute scale) for cubic pyrite, $\mathrm{FeS}_{2}$, as a function of the photon energy in comparison to full-potential (FP) and muffin-tin (MT) calculations. $\operatorname{Re}\left(f_{\|}-f_{\perp}\right)$ (top) and $\operatorname{Im}\left(f_{\|}-f_{\perp}\right)$ (bottom) (Kokubun et al., 2004). tative evaluation of the 'forbidden' reflections using the FDMNES code (Joly, 2001)

Extremely interesting is the observation of the 'forbidden' 010 reflection near the $\mathrm{C} K$ edge in caesium hydrogen phthalate, $\mathrm{C}_{6} \mathrm{H}_{4}(\mathrm{COOH}) \mathrm{COOCs}$, space group Pbca (Okotrub et al., 1998). Owing to the long $b$ axis, $b=25.523 \AA$, it was possible to measure this reflection even at an energy as low as $0.2838 \mathrm{keV}$.

The high sensitivity of the tensorial ASF to displacements of both the resonant atom and neighbouring atoms can also give rise to additional reflections in resonant X-ray diffraction by incommensurately modulated crystals. It has been shown that in that case there is the possibility of both 'forbidden' main reflections and 'forbidden' satellites (Ovchinnikova \& Dmitrienko, 1999a,b). Preliminary observations of 'forbidden' reflections in $\mathrm{Ca}_{2} \mathrm{CoSi}_{2} \mathrm{O}_{7}$ (Co-Åkermanite) have been reported by Soejima et al. (2003).

Another interesting example was found in chiral smectic liquid crystals with an incommensurate spiral structure (Mach et al., 1998; Levelut \& Pansu, 1999; Gorkunov et al., 1999; Matkin et al., 2000; Hirst et al., 2002); the issue was recently surveyed by Clegg (2005). The observations of 'forbidden' reflections near the $\mathrm{S}$ and $\mathrm{Se} K$ edges provided the first direct evidence of distinct periodicities in the structures of several chiral smectic phases. 'Forbidden' reflections of this kind predicted for icosahedral quasicrystals with $5_{1}, 5_{2}, 5_{3}$ and $5_{4}$ screw axes (Dmitrienko, 1989) have not yet been observed.

As for the allowed reflections, the information usually retrievable from 'forbidden' reflections is limited by the socalled phase problem: only the structure-dependent intensity of a reflection is measured whereas the phase information gets lost (including the phase of the tensorial ASF). A well known method to nevertheless obtain phase information (see the survey by Weckert \& Hümmer, 1997) is the interference of a 'forbidden' reflection with multiple-wave contributions from non-forbidden reflections (also called Renninger reflections). Renninger reflections occur for certain $\psi$ angles when the crystal is rotated about the scattering vector. Usually they are considered as unwanted effects hampering the determination of the true $\psi$ dependence of a 'forbidden' reflection (Fig. 1), however careful analysis of the interference regions allows for determining the unknown phases of 'forbidden' reflections (Kokubun, Ishida \& Dmitrienko, 1998) because the phase of the multiple-wave contribution is usually known (more precisely, it allows for obtaining the so-called structure invariants). Until now, there have only been two studies of this type: (i) the determination of the phase of the 006 reflection in Ge at a fixed X-ray energy (Lee et al., 2001), and (ii) the measurement of the real and imaginary parts of the anisotropy as functions of the X-ray energy near the Fe absorption edge in $\mathrm{FeS}_{2}$ (Kokubun et al., 1997, 2004), see Fig. 3. From Fig. 3, one also gets an impression of the quality of modern ASF calculations using the muffin-tin (MT, Vedrinskii et al., 1992) and full-potential (FP, Taillefumier et al., 2002) methods.

A very interesting problem is the interference between magnetic and anisotropic tensor susceptibility reflections (Tsuji et al., 1996; Ovchinnikova \& Dmitrienko, 1997, 2000; 
Collins et al., 2001; Ji et al., 2003; Kokubun, Watanabe et al., 2005) but this is beyond the scope of the present paper.

\section{Partial structure determination and phase information}

Since the 'forbidden' reflection intensities are exclusively due to scattering from the absorbing 'edge atoms', they are signals from their partial structure and, since this selective scattering is undisturbed by the non-resonant scattering 'rest structure', independent of its size and complexity, it can be used as a probe for partial structure analysis, i.e. to locate the 'edge atom'. Templeton \& Templeton (1986) showed in agreement with a suggestion by Dmitrienko (1983) that their first measurements of 'forbidden' axial reflections $00 l, l=2 n+1$ (up to order 11), in $\mathrm{NaBrO}_{3}$ allowed for the determination of the Br-atom position on the threefold axis: a value of $x(\mathrm{Br})=$ 0.408 (1) was derived by least squares from the data for 'forbidden' reflections [true $x(\mathrm{Br})=0.40640(3)$ ]. Later a method was suggested to derive from these data the correct phases for the allowed reflections $00 l, l=4 n+2$ (Templeton $\&$ Templeton, 1987). A more complicated example is provided by $\mathrm{Ba}\left(\mathrm{BrO}_{3}\right)_{2} \cdot \mathrm{H}_{2} \mathrm{O}$ (Templeton \& Templeton, 1992), for which 11 'forbidden' reflections served to derive $x(\mathrm{Br})=$ 0.249 (1) and $z(\mathrm{Br})=0.557$ (1) (true $x=0.2510$ and $z=0.5574$ ).

The way in which the azimuthal intensity variations $I(\psi)$ of 'forbidden' reflections in monoclinic, orthorhombic and tetragonal structures depend on the "edge atom's" position, i.e. reflect the partial structure projection on the respective axes, has been worked out by Kirfel \& Morgenroth (1993), Kirfel (1994) and Morgenroth et al. (1994a,b). The information given therein is derived under the assumptions that (i) the incident radiation is purely $\sigma$ polarized, (ii) the scattering plane is vertical, and (iii) there is only one 'edge atom' in the asymmetric unit. Accordingly, the $\boldsymbol{\sigma}$ - and $\boldsymbol{\pi}$-polarized contributions of the scattered radiation are compiled as functions of the relevant scattering-factor-tensor off-diagonal elements $f_{i j}$ and the crystal azimuthal setting $\psi$. The use of this information for partial structure determination was first discussed for $\mathrm{LiHSeO}_{3}$ crystallizing in space group $P 2_{1} 2_{1} 2_{1}$ (Kirfel et al., 1990; Kirfel \& Lippmann, 1992; Kirfel \& Petcov, 1991). For this non-centrosymmetric case, it can be shown that the scattered radiation of a 'forbidden' $00 l$ reflection $(l=2 n+1)$ is completely $\pi$ polarized and that

$$
I(00 l ; \psi)=K \cos ^{2} \theta\left[A \sin ^{2} \psi+B \cos ^{2} \psi+D \sin 2 \psi\right]
$$

with $A=\left|f_{23}\right|^{2} c_{l z}^{2}, B=\left|f_{13}\right|^{2} s_{l z}^{2}, D=\left(f_{13}^{\prime} f_{23}^{\prime \prime}-f_{23}^{\prime} f_{13}^{\prime \prime}\right) c_{l z} s_{l z}$ and $c_{l z}=\cos 2 \pi l z, s_{l z}=\sin 2 \pi l z ; K$ is the scale factor. Then, using the intensity values at $\psi=0$ and $\pi / 2$ and calculating, for each pair of reflections, one has that the experimental quantity

$$
Q\left(z, l_{1}, l_{2}\right)=\frac{I\left(00 l_{1} ; 0\right) I\left(00 l_{2} ; \pi / 2\right)}{I\left(00 l_{1} ; \pi / 2\right) I\left(00 l_{2} ; 0\right)} \frac{\tan ^{2} 2 \pi l_{1} z}{\tan ^{2} 2 \pi l_{2} z}
$$

eliminates everything but the geometric factors. Thus, the method of deriving an atom coordinate is based on the ratio of any two reflections of the same kind, which cancels the scaling as well as the unknown magnitude of the resonance effect so that the mere excitation of 'forbidden' reflections allows for deriving $Q\left(z, l_{i}, l_{j}\right)$. Then, the combination of at least three such ratios yields a common indication for the possible $z$ coordinate(s), in the given case, a value between 0 and 0.25 from which the symmetry-equivalent $z$ coordinates can be calculated. Since corresponding relations hold for the 'forbidden' $h 00$ and $0 k 0$ reflections, respectively, one obtains altogether 16 possible $(x, y, z)$ sets, one of which approximates the correct 'edge-atom' position. In a more general treatment (Kirfel \& Petcov, 1992), the intensity values must be corrected by a factor accounting for anisotropic absorption. However, as discussed by Kirfel \& Petcov (1992), neglecting these corrections is not likely to compromise the method. Results obtained for the $y$ and $z$ coordinates of $\mathrm{Se}$ in $\mathrm{LiHSeO}_{3}$ were 0.150 (5) and $0.235(15)$, respectively, as compared with the true coordinates $y=0.14709$ (2) and $z=0.23316$ (4). Other examples of similar edge-atom locations are $\mathrm{K}_{2} \mathrm{Co}\left(\mathrm{SO}_{4}\right)_{2} \cdot 6 \mathrm{H}_{2} \mathrm{O}, P 2_{1} / c$, $y(\mathrm{Co})=0.014$ (true 0.0) (Fischer et al., 1996a), $\mathrm{CuSeO}_{3} \cdot 2 \mathrm{H}_{2} \mathrm{O}$, $P 2{ }_{1} 2_{1} 2_{1}, z(\mathrm{Cu})=0.0461$ (true 0.043) (Kirfel \& Lippmann, 1994), $\mathrm{Na}_{2}\left[\mathrm{Fe}(\mathrm{CN})_{5} \mathrm{NO}\right] \cdot 2 \mathrm{H}_{2} \mathrm{O}$, Pnnm, $x(\mathrm{Fe})=-0.005$ (true $0.014), y(\mathrm{Fe})=0.215$ (true 0.220), $z(\mathrm{Fe})=0.02$ (true 0.0) (Kirfel et al., 1995b) and finally $\mathrm{KH}_{3}\left(\mathrm{SeO}_{3}\right)_{2}, P b c n, x(\mathrm{Se})=$ 0.150 (true 0.1514), $y(\mathrm{Se})=0.185$ (true 0.188 ) and $z(\mathrm{Se})=0.22$ (true 0.2125) (Fischer \& Kirfel, 1998). In all these cases, the absolute differences between the experimental and true atom positions were less than $0.1 \AA$.

So far, the method has been successfully tested and verified on samples with just one 'edge atom' in the asymmetric unit. However, it should also be applicable to more atoms if different 'edge elements' are involved. One interesting case would be a large organic molecular structure with two or more different 'edge atoms' per asymmetric unit. Knowing their positions in the molecule, one should be able to confine the molecule's position and orientation to a small number of possibilities that can be further explored in combination with other methods of structure determination. As the method can distinguish between neighbouring elements in the periodic system (e.g. $\mathrm{Fe}, \mathrm{Co}, \mathrm{Ni})$ as can neutron diffraction, it has the advantage that one needs only access to a synchrotronradiation source. Hence, there is the option to collect reflection data and do partial structure determination on the same sample in one experiment at a given source. However, to that purpose, we do need more experience as to whether or not we can obtain reasonable 'resonant scattering' information from a sample suitable for conventional data acquisition, e.g. from a small crystal sphere bathing in the beam. So far, practically all resonant experiments have been done, with obvious experimental advantages and because other goals were aimed at, on extended sample faces being only partially illuminated by the beam. A few successful experiments on small crystals like that by Templeton \& Templeton (1997) would make progress in the field.

Except for a study on $\mathrm{VOSO}_{4} \cdot 5 \mathrm{H}_{2} \mathrm{O}$ by Templeton \& Templeton (1991), little use has been made so far of the information contained in the intensity variations $I(h k l ; \psi)$ of the allowed reflections, which are generally also affected by anisotropic resonant scattering. However, knowing for 
example from the 'forbidden' reflections the partial structure of the 'edge atom' in a centrosymmetric structure (or a centrosymmetric projection), i.e. knowing $r_{a}$, one should also be able to use the intensity patterns of allowed central lattice row reflections for determining their structure-factor signs relative to each other, even if the contribution of the partial structure does not dominate. This option has been discussed by Kirfel \& Fischer (1998), successfully applied in a model calculation on $\mathrm{YBa}_{2} \mathrm{Cu}_{3} \mathrm{O}_{7}$ and experimentally tested on $\mathrm{KH}_{3}\left(\mathrm{SeO}_{3}\right)_{2}$ (Kirfel, 1999). Structure-factor relative sign determination is a still rather unexplored method and needs more experimental evidence and verification. At present, it is therefore certainly too early to speculate about its potential with respect to routine structure determination but, if (see above) one could show that measuring on a 'normal' crystal is able to yield on-line relative sign information, this would be a step forward to direct structure imaging.

\section{Beyond the dipole-dipole approximation}

Some screw-axis/glide-plane reflections remain 'forbidden' in the $d d$ approximation, for instance, the reflections with the scattering vector along the threefold rotation axes in cubic, rhombohedral and hexagonal crystals (Dmitrienko, 1983). However, they can be excited in cases where there are $d q$ and/or $q q$ contributions to the ASF [equation (2)]. The first 'forbidden' reflection of this type was found by Finkelstein $e t$ al. $(1992,1994)$ in the pre- $K$-edge peak of $\mathrm{Fe}$ in hematite, $\alpha-\mathrm{Fe}_{2} \mathrm{O}_{3}$ (reflection 0003 or 111 in hexagonal or rhombohedral settings, respectively; the rhombohedral one is used here). Since the Fe atoms are on the threefold axes, their $f$ tensors are uniaxial (in the $d d$ approximation) and oriented along the threefold axes so that they have the same form for all the atoms and hence yield zero structure factors for the glideplane reflections $h h h, h=2 n+1$. In contrast, the $d q$ and $q q$ $f$ tensors possess different signs of some tensor components of the glide-plane-related atoms, and just the components $f_{z x y}^{d q a}$ and $f_{x x x z}^{q q}$ give non-zero contributions to the structure factors of the 'forbidden' reflections (Dmitrienko \& Ovchinnikova, 2001):

$$
\begin{aligned}
\boldsymbol{\sigma} \widehat{F}(h h h ; h=\text { odd }) \boldsymbol{\pi}= & -8 i|\mathbf{k}| f_{z x y}^{d q a} \sin (6 \pi h x) \cos ^{2} \theta \\
& +4 \cos (6 \pi h x) f_{x x x z}^{q q} \mathbf{k}^{2} \cos ^{3} \theta \sin 3 \psi \\
\boldsymbol{\pi}^{\prime} \widehat{F}(h h h ; h=\text { odd }) \boldsymbol{\sigma}= & 8 i|\mathbf{k}| f_{z x y}^{d q a} \sin (6 \pi h x) \cos ^{2} \theta \\
& +4 \cos (6 \pi h x) f_{x x x z}^{q q} \mathbf{k}^{2} \cos ^{3} \theta \sin 3 \psi .
\end{aligned}
$$

A pure $q q$ transition results in a sixfold symmetry of the azimuthal dependence $\left(\propto \sin ^{2} 3 \psi\right)$ of the $h h h$ intensity as was initially claimed by Finkelstein et al. $(1992,1994)$, but interference with a (azimuthally independent) $d q$ contribution makes the azimuthal pattern threefold symmetric. This threefold symmetry (Fig. 4) was recently observed by Watanabe et al. (2001), Kokubun \& Ishida (2003) and Kokubun, Watanabe et al. (2005), and it is confirmed by computer simulations (Di Matteo et al., 2003). Notice that the difference between the $\sigma \rightarrow \pi^{\prime}$ and $\pi \rightarrow \sigma$ channels follows from the antisymmetric $d q$ contribution. It can be shown (Kokubun, Sawai et al., 2005) that the pre-edge peak in anatase (Fig. 2) is induced by symmetric and antisymmetric $d q$ terms (no $d d$ and $q q$ contributions).

There is interesting physics behind the antisymmetric $d q$ terms: they relate to a local chirality of the Fe atoms. Indeed, two atoms per unit cell have right-hand environments whereas the two others, related to the first two by inversion, possess left-hand environments. If there was only one sort of atom, say right-hand, then the $d q$ terms would lead to circular dichroism but, in the real structure, this dichroism is exactly compensated by the other sort of atom. Only the 'forbidden' reflections allow for detecting the right-left difference between the two kinds of atoms whereas the conventional X-ray optical activity is rather difficult to observe (Siddons et al., 1990; Alagna et al., 1998; Goulon et al., 1998, 2000).

Another situation with vanishing $d d$ contribution arises when atoms are in special positions of high site symmetry (Templeton, 1998), as for instance in the crystal structure of germanium with $\mathrm{Ge}$ site symmetry $\overline{4} 3 \mathrm{~m}$. In this case, 'forbidden' reflections $0 k l, k+l=4 n+2$, can be excited owing to the tetrahedral anisotropy described by the symmetric third-rank tensor $f_{j m n}^{d q s}$ with only one type of nonzero component: $f_{x y z}^{d q s}=f_{y x z}^{d q s}=f_{y z x}^{d q s} f_{z y x}^{d q s}=f_{z x y}^{d q s}=f_{x z y}^{d q s}$ (Templeton \& Templeton, 1994). The corresponding structure factor is given by

$$
\widehat{F}(0 k l ; k+l=4 n+2)=8 f_{x y z}^{d q s} \frac{2 \pi}{a}\left(\begin{array}{ccc}
0 & l & k \\
l & 0 & 0 \\
k & 0 & 0
\end{array}\right) .
$$

Templeton \& Templeton (1994) observed the azimuthal dependence of the reflections 280 and 482 in accordance with equation (15). However, later it was found that exactly the same dependence could be induced by thermal motion of atoms even in the $d d$ approximation (see §8) and the latter effect is predominant. Templeton \& Templeton (1994) observed similar reflections, 140 and 340 , in $\mathrm{K}_{2} \mathrm{CrO}_{4}$ (space group Pnma), however their temperature dependence has not yet been studied. Fig. 2 shows the $d q$ effect in the pre-edge peak of a $\mathrm{TiO}_{2}$ anatase crystal (space group $\mathrm{I}_{1} /$ amd, site

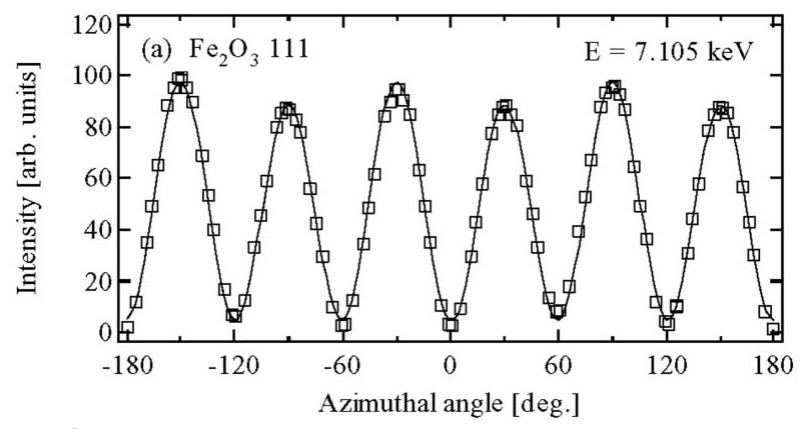

Figure 4

The azimuthal angle dependence of the reflection 111 in hematite at the pre-edge peak energy $E=7105 \mathrm{eV}$ (Kokubun, Watanabe et al., 2005). The threefold symmetry of the plot is evidence for the local chirality of the Fe atoms. 
symmetry $\overline{4} m 2$ ). It is shown (Kokubun, Sawai et al., 2005) that this peak is caused by both a symmetric and an antisymmetric $d q$ contribution, $d d$ and $q q$ transitions are not involved. Here, it should be noted that the Ti site symmetry allows also for the thermal-motion-induced $d d$ effect but thermal motion cannot simulate an antisymmetric $d q$ contribution as considered above for hematite.

In the case that symmetry simultaneously allows for $d d, d q$ and/or $q q$ contributions for the same reflection, the different contributions can be distinguished by polarization, azimuthal and spectral behaviour. For the Fe-atom pre-edge peak, evidence for the $d q$ effect was found this way in $\mathrm{Fe}_{3} \mathrm{O}_{4}$ (Kirfel et al., 1995a; Fischer, Krane \& Kirfel, 1996; García et al., 2000); moreover, comparing the measurements of different reflections, Kanazawa et al. (2002) have found some $d q$ resonance contributing even into the main peak. Kokubun, Nagano et al. (1998) studied the azimuthal dependence of the energy spectra of the pre-edge peaks in pyrite and attributed the peaks to the $q q$ effect.

\section{Thermal-motion-induced and defect-induced reflections}

A new dimension in the field was added in 1999 with a publication by Dmitrienko, Ovchinnikova \& Ishida, who contended that the excitation of 'forbidden' reflections can also be induced by thermal vibrations [these will be referred to as thermal-motion-induced (TMI) reflections]. Later, a similar effect was also predicted for randomly distributed point defects (Dmitrienko \& Ovchinnikova, 2000). Owing to thermal motion, each atom continuously experiences displacements from its average position into places with lower symmetry (in general with point symmetry 1) so that, according to the adiabatic approximation, the electron states are temporarily distorted to a symmetry lower than that of the atom's average site. Since the atomic vibrations are of the order of $10^{4}$ times slower than the scattering process, the radiation 'snapshots' an 'edge-atom' scattering-factor tensor that is no longer constrained to the higher symmetry of the time-averaged atom position. In order to assess the properties of a 'forbidden' reflection, it is therefore necessary to calculate the structure-factor tensor as an average over all temporary configurations by adding to the scattering-factor tensor for the atom at rest a term that accounts for the displacements, relative to each other, of the 'edge atom' itself and its nearest neighbours. This is achieved by introducing a correlation function which contains the eigenfrequencies and eigenvectors of optical phonons so that the structure factor becomes sensitive to phonon properties (even in the harmonic approximation).

The experimental validation of the TMI concept necessarily requires the observation of a temperature-dependent 'forbidden' reflection intensity that cannot be explained by the Debye-Waller factor of the crystal structure. A perfect candidate was found in germanium for which $I(006)$ was expected to decrease with increasing temperature if solely caused by a $d q$ transition. Two independent experiments
(Kokubun et al., 2001; Kirfel et al., 2002b) revealed exactly the opposite: between 30 and $700 \mathrm{~K}, I(006)$ increased by a factor of 30 (at the resonant energy). This anomalous temperature dependence was initially described in terms of a relatively simple autocorrelation model but significant improvement was later obtained upon introducing cooperative vibrations of the atoms. The spectral and polarization properties of the 006 reflection at ambient conditions were studied in detail by Detlefs (2004).

While in germanium in spite of the large intensity gain the form of the resonance, i.e. the energy spectra, did not significantly change with temperature, the observed variation of the 'forbidden' reflection 115 in hexagonal wurtzite-type $\mathrm{ZnO}$ (space group $P 6_{3} m c$ ) was found to be accompanied by changes in the resonance profile curves (Collins et al., 2003). These observations can be rationalized in terms of an interference of the $d d$ TMI effect with an electronic $d q$ transition which has very different energy profiles. Collins et al. (2003) were able to model the complicated pattern of $I(115 ; E, T)$ by a function

$$
I(E, T)=\left|A(E) \exp i \phi(E)+B(E) \operatorname{coth}\left(\frac{\hbar \omega_{0}}{2 k T}\right)\right|^{2} \exp (-2 M),
$$

which describes the effects of a complex solely energy dependent transition structure factor and a term that is both energy and temperature dependent for isotropic atomic displacements corresponding to a low-lying phonon branch with characteristic frequency $\omega_{0}$. Inclusion of the possibility that both terms suffer the same thermal 'fall-off' caused by the Debye-Waller factor $[\exp (-2 M)]$ allowed the observations to be remarkably well fitted from 59 to $800 \mathrm{~K}$.

Thus, the phenomenological fitting gives a good explanation of the temperature effects, both for $\mathrm{Ge}$ and for $\mathrm{ZnO}$, but it cannot describe the energy dependence. In recent contributions (Oreshko et al., 2004; Dmitrienko et al., 2004; Ovchinnikova, Dmitrienko et al., 2005; Ovchinnikova, Oreshko et al.2005), numerical calculations were performed with the help of the codes developed by Vedrinskii et al. (1992), Joly (2001) and Taillefumier et al. (2002). These calculations, which allow for quantitative fitting of the experimental data, show the high sensitivity of ASF to atomic displacements. For Ge, such numerical calculations gave a satisfactory modelling of the 006 forbidden-reflection spectrum, its thermal growth, the behaviour of the line width and peak position at different temperatures. In $\mathrm{ZnO}$, it was shown that a more detailed analysis based on three different phonon branches may be needed for a better description of the energy spectra at different temperatures.

In view of the results obtained in the past three years, it seems justified to infer that TMI anisotropy is a rather general phenomenon because there is no reason why it should be absent in other structures. TMI can, for example, be the reason for the different temperature dependencies of the 'forbidden' reflections 100 and 300 on one hand and the allowed 200 on the other as observed in a low-temperature study on cuprite (Kirfel \& Krane, 1999). An obvious growth of the 002 reflection intensity of magnetite has been observed by Subías, 
García et al. (2004) in the temperature range from 300 to $900 \mathrm{~K}$. Thus, a full characterization of the origin and nature of a 'forbidden' reflection requires measurements of the intensity dependence on not only energy and azimuthal setting but also temperature. For the azimuthal behaviour, single-crystal diffraction is an indispensable prerequisite, but studies of the other dependencies may be alleviated by using powder samples provided the reflections of interest are not contaminated by overlapping. This possibility has been tested in an early experiment on cuprite (Kirfel, Eichhorn \& Wroblewski, 1988).

\section{Conclusions}

Summing up, the field of resonant scattering with emphasis on studying 'forbidden' reflections as sensitive probes of the absorbing atom's environment and partial structure has gained new life with the postulation and proof of the TMI anisotropy of resonant scattering. This is because the phonon time scale is so large that X-ray diffraction 'sees' temporarily distorted electronic states as stationary ones.

One of the key problems is the experimental determination of 'forbidden' reflections and the local anisotropy on an absolute scale because the multiple-wave solution of the phase problem is extremely time consuming. In principle, this aspect of information about local anisotropy of resonant scattering can be obtained from a structure analysis including the energy dependence of the non-forbidden reflections. This method has been developed and widely used by Templeton \& Templeton: $\mathrm{NaUO}_{2}\left(\mathrm{C}_{2} \mathrm{H}_{3} \mathrm{O}_{2}\right)_{3}$, space group $P 2_{1} 3$ (1982); $\mathrm{K}_{2} \mathrm{PtCl}_{4}$, $P 4 / m m m ~(1985 b) ; \mathrm{C}_{6} \mathrm{H}_{12} \mathrm{~N}_{2} \mathrm{O}_{4} \mathrm{Se} \quad\left(P 4_{3} 2_{1} 2\right)$ (1988); $\mathrm{LiIO}_{3}$ $\left(P 6_{3} 22\right)(1989 a) ; \mathrm{C}_{16} \mathrm{H}_{14} \mathrm{BrNO}(P \overline{1})(1995)$; and it is even feasible to distinguish the resonant scattering of the same element in non-equivalent sites, as in $\mathrm{Cs}_{2}\left[\mathrm{AuCl}_{2}\right]\left[\mathrm{AuCl}_{4}\right]$ (Wilkinson et al., 1995) and in $\mathrm{NdNiO}_{3}$ (Lorenzo et al., 2005). This method was called DAFS (diffraction anomalous fine structure), see, for example, a survey of Hodeau et al. (2001). As a possible further development of the DAFS method, it would be particularly interesting to use an appropriate polarization analysis technique, e.g. to measure allowed reflections with crossed polarizers (in $\sigma$-to- $\pi$ or $\pi$-to- $\sigma$ mode). However, since some tensor contributions to ASF can be determined only from 'forbidden' reflections, their investigation is still indispensable; an example is the third-rank-tensor contribution in Ge. Especially interesting would be the application of this technique to biological molecules; for recent developments see the paper of Bricogne et al. (2005).

Another key problem is the access to synchrotron radiation because the experiments are rather time consuming, particularly in the initial steps of establishing the resonance(s), proving the excitation(s) of 'forbidden' reflections and optimizing the experimental set-up. Owing to limited beam times, it is often impossible to use a successfully working set-up for a time sufficient to obtain more and/or higher-quality data or to tackle open questions. Instead, in each experimental session, valuable time is spent in the set-up stage, which could be saved were a longer-lasting beam-time allocation available. From a practical point of view, this explains to a great extent the relatively slow progress in the field.

This work is partly supported by grant INTAS 01-0822. The authors are grateful to J. Kokubun for numerous discussions and his help in the manuscript preparation as well as to S. P. Collins, J. García, J. E. Lorenzo, G. Subías, D. H. Templeton and referees for numerous helpful remarks and suggestions. KI deeply acknowledges the staff of the Photon Factory at KEK where all of the presented measurements of his group were carried out over the last 15 years. For most of the experimental studies carried out at HASYLAB, AK gratefully acknowledges the financial support by the German Minister for Research and Education granted over a 12-year period.

\section{References}

Alagna, L., Prosperi, T., Turchini, S., Goulon, J., Rogalev, A., GoulonGinet, C., Natoli, C. R., Peacock, R. D. \& Stewart, B. (1998). Phys. Rev. Lett. 80, 4799-4802.

Ankudinov, A. L., Ravel, B., Rehr, J. J. \& Conradson, S. D. (1998). Phys. Rev. B, 58, 7565-7576.

Belyakov, V. A. (1975). Sov. Phys. Usp. 18, 267-299. Translated from Usp. Fiz. Nauk, 115, 553-601.

Belyakov, V. A. \& Dmitrienko, V. E. (1989). Sov. Phys. Usp. 32, 697-719. Translated from Usp. Fiz. Nauk, 158, 672-721.

Belyakov, V. A., Dmitrienko, V. E. \& Orlov, V. P. (1979). Sov. Phys. Usp. 22, 64-88. Translated from Usp. Fiz. Nauk, 127, 221-261.

Benfatto, M. \& Felici, R. (2001). Phys. Rev. B, 64, 115410-1115410-10.

Benfatto, M., Joly, Y. \& Natoli, C. R. (1999). Phys. Rev. Lett. 83, 636-639.

Blume, M. (1994). Resonant Anomalous X-ray Scattering, edited by G. Materlik, C. J. Spark \& K. Fisher, pp. 495-515. Amsterdam: North-Holland.

Bricogne, G., Capelli, S. C., Evans, G., Mitschler, A., Pattison, P., Roversi, P. \& Schiltz, M. (2005). J. Appl. Cryst. 38, 168-182.

Brouder, C. (1990). J. Phys. Condens. Matter, 2, 701-738.

Carra, P. \& Thole, B. T. (1994). Rev. Mod. Phys. 66, 1509-1515.

Clegg, P. S. (2005). Acta Cryst. A61, 112-121.

Collins, S. P., Laundy, D., Dmitrienko, V. E., Mannix, D. \& Thompson, P. (2003). Phys. Rev. B, 68, 064110-1-064110-4.

Collins, S. P., Laundy, D. \& Stunault, A. (2001). J. Phys. Condens. Matter, 13, 1891-1905.

Dawson, B. (1975). Advances in Structure Research by Diffraction Methods, edited by W. Hoppe \& R. Mason, Vol. 6, pp. 1-250. Oxford: Pergamon Press.

Detlefs, C. (2004). Physica (Utrecht), B345, 45-48.

Di Matteo, S., Joly, Y., Bombardi, A., Paolasini, L., de Bergevin, F. \& Natoli, C. R. (2003). Phys. Rev. Lett. 91, 257402-1-257402-4.

Dmitrienko, V. E. (1983). Acta Cryst. A39, 29-37.

Dmitrienko, V. E. (1984). Acta Cryst. A40, 89-95.

Dmitrienko, V. E. (1989). Pis'ma Zh. Eksp. Teor. Fiz. 50, 153-156. Engl. transl: JETP Lett. 50, 171-174.

Dmitrienko, V. E. \& Ovchinnikova, E. N. (2000). Acta Cryst. A56, 340-347.

Dmitrienko, V. E. \& Ovchinnikova, E. N. (2001). Acta Cryst. A57, 642-648.

Dmitrienko, V. E. \& Ovchinnikova, E. N. (2003). Crystallogr. Rep. 48, Suppl. 1, S52-S68. Translated from Kristallografiya, 48, Suppl., S59-S77.

Dmitrienko, V. E., Ovchinnikova, E. N. \& Ishida, K. (1999). Pis'ma Zh. Eksp. Teor. Fiz. 69, 885-889. Engl. transl: JETP Lett. 69, 938-942. 
Dmitrienko, V. E. Ovchinnikova, E. N., Ishida, K., Kokubun, J., Kirfel, A., Collins, S. P., Laundy, D., Oreshko, A. P. \& Cabaret, D. (2004). Phys. Status Solidi C, 1, 3081-3084.

Eichhorn, K., Kirfel, A. \& Fischer, K. (1988). Z. Naturforsch. Teil A, 43, 391-392.

Elfimov, I. S., Anisimov, V. I. \& Sawatzky, G. A. (1999). Phys. Rev. Lett. 82, 4264-4267.

Finkelstein, K. D., Hamrick, M. \& Shen, Q. (1994). Resonant Anomalous X-ray Scattering, edited by G. Materlik, C. J. Spark \& K. Fisher, pp. 91-97. Amsterdam: North-Holland.

Finkelstein, K. D., Shen, Q. \& Shastri, S. (1992). Phys. Rev. Lett. 69, 1612-1615.

Fischer, R. \& Kirfel, A. (1997). HASYLAB Annual Report, pp. 339-340.

Fischer, R. \& Kirfel, A. (1998). HASYLAB Annual Report, pp. 979-980.

Fischer, R., Kirfel, A. \& Morgenroth, W. (1996a). HASYLAB Annual Report, pp. 652-653.

Fischer, R., Kirfel, A. \& Morgenroth, W. (1996b). HASYLAB Annual Report, pp. 654-655.

Fischer, R., Krane, H.-G. \& Kirfel, A. (1996). HASYLAB Annual Report, pp. 650-651.

García, J., Sánchez, M. C., Blasco, J., Subías. G. \& Proietti, M. G. (2001). J. Phys. Condens. Matter, 13, 3243-3256.

García, J. \& Subías, G. (2004). J. Phys. Condens. Matter, 16, R145-R178.

García, J., Subías, G., Proietti, M. G., Blasco, J., Renevier, H., Hodeau, J. L. \& Joly, Y. (2001). Phys. Rev. B, 63, 054110-1-054110-6.

García, J., Subías, G., Proietti, M. G., Renevier, H., Joly, Y., Hodeau, J. L., Blasco, J., Sánchez, M. C. \& Bérar, J. F. (2000). Phys. Rev. Lett. 85, 578-581.

Gibbs, D., Moncton, D. E. \& D'Amico, K. L. (1985). J. Appl. Phys. 57, 3619-3622.

Gorkunov, M. V., Pikin, S. A. \& Haase, W. (1999). JETP Lett. 69, 243-249.

Goulon, J., Goulon-Ginet, C., Rogalev, A., le Benayoun, G., Brouder, C. \& Natoli, C. R. (2000). J. Synchrotron Rad. 7, 182-188.

Goulon, J., Goulon-Ginet, C., Rogalev, A., Gotte, V., Malgrange, C., Brouder, C. \& Natoli, C. R. (1998). J. Chem. Phys. 108, 6394-6403.

Hagiwara, K., Kanazawa, M., Horie, K., Kokubun, J. \& Ishida, K. (1999). J. Phys. Soc. Jpn, 68, 1592-1597.

Hannon, J. P., Trammell, G. T., Blume, M. \& Gibbs, D. (1988). Phys. Rev. Lett. 61, 1245-1248.

Herrero-Martín, J., García, J., Subías, G., Blasco, J. \& Sánchez, M. C. (2004). Phys. Rev. B, 70, 024408-1-024408-10.

Hirst, L. S., Watson, S. J., Gleeson, H. F., Cluzeau, P., Barois, P., Pindak, R., Pitney, J., Cady, A., Johnson, P. M., Huang, C. C., Levelut, A.-M., Srajer, G., Pollmann, J., Caliebe, W., Seed, A. et al. (2002). Phys. Rev. E, 65, 041705-1-041705-10.

Hock, A., Kirfel, A. \& Lippmann, T. (1995). HASYLAB Annual Report, pp. 365-366.

Hock, A., Lippmann, T. \& Kirfel, A. (1995). HASYLAB Annual Report, pp. 367-368.

Hodeau, J.-L., Favre-Nicolin, V., Bos, S., Renevier, H., Lorenzo, E. \& Berar, J.-F. (2001). Chem. Rev. 101, 1843-1867.

International Tables for Crystallography (1996). Vol A, edited by Th. Hahn. Dordrecht: Kluwer Academic Publishers.

International Tables for Crystallography (2003). Vol. D, edited by A. Authier. Dordrecht: Kluwer Academic Publishers.

Ishihara, S. \& Maekawa, S. (2002). Rep. Prog. Phys. 65, 561-598.

Izyumov, Yu. A., Naish, V. E. \& Ozerov, R. P. (1991) Neutron Diffraction of Magnetic Material. New York: Consultants Bureau, Plenum Publishing Corporation. Translated from Neitrony $i$ Tverdoe Telo. Vol. 2. Neitronografiya Magnetikov. Moskow: Atomizdat.

Ji, S., Song, C., Koo, J., Lee, K.-B., Park, Y. J., Kim, J. Y., Park, J.-H., Shin, H. J., Rhyee, J. S., Oh, B. H. \& Cho, B. K. (2003). Phys. Rev. Lett. 91, 257205-1-257205-4.
Joly, Y. (2001). Phys. Rev. B, 63, 125120-1-125120-10. FDMNES code: http://www-cristallo.grenoble.cnrs.fr/simulation.

Joly, Y., Grenier, S. \& Lorenzo, J. E. (2003). Phys. Rev. B, 68, 104412-1-104412-4.

Kanazawa, M., Hagiwara, K., Kokubun, J. \& Ishida, K. (2002). J. Phys. Soc. Jpn, 71, 1765-1770.

Kirfel, A. (1994). Resonant Anomalous X-ray Scattering, edited by G. Materlik, C. J. Spark \& K. Fisher, pp. 231-256. Amsterdam: North-Holland.

Kirfel, A. (1999). Unpublished results.

Kirfel, A. \& Eichhorn, K. (1988). HASYLAB Annual Report, pp. 265-266.

Kirfel, A. \& Eichhorn, K. (1989). HASYLAB Annual Report, pp. 383-384.

Kirfel, A., Eichhorn, K. \& Wroblewski, T. (1988). HASYLAB Annual Report, pp. 267-268.

Kirfel, A. \& Fischer, R. (1998). Z. Kristallogr. 213, 337-342.

Kirfel, A., Grybos, J. \& Dmitrienko, V. E. (2002a). HASYLAB Annual Report, pp. 957-958.

Kirfel, A., Grybos, J. \& Dmitrienko, V. E. (2002b). Phys. Rev. B, 66, 165202-1-165202-7.

Kirfel, A. \& Krane, H.-G. (1999). HASYLAB Annual Report, pp. $567-568$.

Kirfel, A. \& Lippmann, T. (1992). HASYLAB Annual Report, pp. 367-368.

Kirfel, A. \& Lippmann, T. (1994). HASYLAB Annual Report, pp. 553-554.

Kirfel, A., Lippmann, T. \& Morgenroth, W. (1995a). HASYLAB Annual Report, pp. 371-372.

Kirfel, A., Lippmann, T. \& Morgenroth, W. (1995b). HASYLAB Annual Report, pp. 373-374.

Kirfel, A. \& Morgenroth, W. (1993). Acta Cryst. A49, 35-45.

Kirfel, A. \& Petcov, A. (1988). HASYLAB Annual Report, pp. 269-270.

Kirfel, A. \& Petcov, A. (1989). HASYLAB Annual Report, pp. 385-386.

Kirfel, A. \& Petcov, A. (1991). Z. Kristallogr. 195, 1-15.

Kirfel, A. \& Petcov, A. (1992). Acta Cryst. A48, 247-259.

Kirfel, A., Petcov, A. \& Eichhorn, K. (1990). HASYLAB Annual Report, pp. 545-546.

Kirfel, A., Petcov, A. \& Eichhorn, K. (1991). Acta Cryst. A47, 180-195.

Kirfel, A., Petcov, A., Fischer, K. \& Eichhorn, K. (1988). HASYLAB Annual Report, pp. 263-264.

Kirfel, A., Petcov, A., Jauch, W. \& Palmer A. (1989). HASYLAB Annual Report, pp. 387-388.

Kokubun, J. \& Ishida, K. (2003). Photon Fact. Act. Rep. 2002, 20A, 21-22.

Kokubun, J. \& Ishida, K. (2005). Private communication.

Kokubun, J., Ishida, K., Cabaret, D., Mauri, F., Vedrinskii, R. V., Kraizman, V. L., Novakovich, A. A., Krivitskii, E. V. \& Dmitrienko, V. E. (2004). Phys. Rev. B, 69, 245103-1-245103-14.

Kokubun, J., Ishida, K. \& Dmitrienko, V. E. (1998). J. Phys. Soc. Jpn, 67, 1291-1295.

Kokubun, J., Kanazawa, M., Ishida, K. \& Dmitrienko, V. E. (2001). Phys. Rev. B, 64, 073203-1-073203-4.

Kokubun, J., Kuribayashi, M., Ishida, K. \& Dmitrienko, V. E. (1997). Photon Fact. Act. Rep. 15B, 7.

Kokubun, J., Nagano, T., Kuribayashi, M. \& Ishida, K. (1998). J. Phys. Soc. Jpn, 67, 3114-3118.

Kokubun, J., Sawai, H., Uehara, M., Ishida, K., Kirfel, A. \& Dmitrienko, V. E. (2005). Unpublished.

Kokubun, J., Watanabe, A., Uehara, M., Ninomiya, Y., Sawai, H., Momozawa, N., Ishida, K. \& Dmitrienko, V. E. (2005). Phys. Rev. $B$. Submitted.

Lee, T. L., Felici, R., Hirano, K., Cowie, B., Zegenhagen, J. \& Colella, R. (2001). Phys. Rev. B, 64, 201316-1-201316-4.

Levelut, A.-M. \& Pansu, B. (1999). Phys. Rev. E, 60, 6803-6815. 
Lippmann, T., Fischer, K. \& Kirfel, A. (1998). J. Appl. Cryst. 31, 94-97.

Lippmann, T. \& Kirfel, A. (1991). HASYLAB Annual Report, pp. 279-280.

Lippmann, T., Kirfel, A. \& Fischer, K. (1992). HASYLAB Annual Report, pp. 365-366.

Lippmann, T., Kirfel, A. \& Fischer, K. (1994). HASYLAB Annual Report, pp. 555-556.

Lippmann, T., Kirfel, A., Morgenroth, W. \& Fischer, K. (1995). HASYLAB Annual Report, pp. 369-370.

Lorenzo, J. E., Hodeau, J. L., Paolasini, L., Lefloch, S., Alonso, J. A. \& Demazeau, G. (2005). Phys. Rev. B, 71, 045128-1-045128-7.

Lovesey, S. W. \& Collins, S. P. (1996) X-ray Scattering and Absorption by Magnetic Materials. Oxford: Clarendon Press.

Mach, P., Pindak, R., Levelut, A.-M., Barois, P., Nguyen, N. T., Huang, C. C. \& Furenlid, L. (1998). Phys. Rev. Lett. 81, 1015-1018.

Materlik, G., Spark, C. J. \& Fisher, K. (1994). Editors. Resonant Anomalous X-ray Scattering. Amsterdam: North-Holland.

Matkin, L. S., Gleeson, H. F., Mach, P., Huang, C. C., Pindak, R., Srajer, G., Pollmann, J., Goodby, J. W., Hird, M. \& Seed, A. (2000). Appl. Phys. Lett. 76, 1863-1865.

Morgenroth, W., Kirfel, A. \& Fischer, K. (1993). HASYLAB Annual Report, pp. 565-566.

Morgenroth, W., Kirfel, A. \& Fischer, K. A. (1994a). Resonant Anomalous X-ray Scattering, edited by G. Materlik, C. J. Spark \& K. Fisher, pp. 257-264. Amsterdam: North-Holland.

Morgenroth, W., Kirfel, A. \& Fischer, K. (1994b). Z. Kristallogr. 209, 124-131.

Nagano, T., Kokubun, J., Yazawa, I., Kurasawa, T., Kuribayashi, M., Tsuji, E., Ishida, K., Sasaki, S., Mori, T., Kishimoto, S. \& Murakami, Y. (1996). J. Phys. Soc. Jpn, 65, 3060-3067.

Natoli, C. R., Benfatto, M., Brouder, C., López, M. F. R. \& Foulis, D. L. (1990). Phys. Rev. B, 42, 1944-1968.

Nazarenko, E., Lorenzo, J. E., Joly, Y., Hodeau, J. L., Mannix, D. \& Marin, C. (2005). In the press.

Ninomiya, Y., Watanabe, A., Kokubun, J. \& Ishida, K. (2001). Photon Fact. Act. Rep. 2000, 18B, 102.

Nye, J. F. (1985). Physical Properties of Crystals. Oxford University Press.

Okotrub, A. V., Belikova, G. S., Turskaya, T. N. \& Mazalov, L. N. (1998). Mater. Res. Soc. Symp. Proc. 524, 161-166.

Oreshko, A. P., Dmitrienko, V. E., Joly, Y., Kirfel, A. \& Ovchinnikova, E. N. (2004). Izv. Russ. Acad. Sci. 68, 578-582. (In Russian.)

Ovchinnikova, E. N. \& Dmitrienko, V. E. (1997). Acta Cryst. A53, 388-395.

Ovchinnikova, E. N. \& Dmitrienko, V. E. (1999a). Acta Cryst. A55, 20-29.

Ovchinnikova, E. N. \& Dmitrienko, V. E. (1999b). J. Alloys Compd 286, 236-240.

Ovchinnikova, E. N. \& Dmitrienko, V. E. (2000). Acta Cryst. A56, $2-10$.

Ovchinnikova, E. N., Dmitrienko, V. E., Ishida, K., Kirfel, A., Collins, S. P., Oreshko, A. P., Cabaret, D., Vedrinskii, R. V., Kraizman, V. L., Novakovich, A. A., Krivitskii, E. V. \& Tolochko, B. P. (2005). Nucl. Instrum. Methods Phys. Res. A, 543, 122-126.

Ovchinnikova, E. N., Oreshko, A. P., Joly, Y., Kirfel, A., Tolochko, B. P. \& Dmitrienko, V. E. (2005). Phys. Scr. T115, 252-254.

Petcov, A. (1989). Internal Report, DESY-F41, 89-08.

Petcov, A., Kirfel, A. \& Fischer, K. (1988). Z. Naturforsch. Teil A, 43, 388-390.

Petcov, A., Kirfel, A. \& Fischer, K. (1990). Acta Cryst. A46, 754-763.

Ramaseshan, S. \& Abrahams, S. C. (1975). Editors. Anomalous Scattering. Copenhagen: Munksgaard.

Renevier, H., Joly, Y., García, J., Subías, G., Proietti, M. G., Hodeau, J. L. \& Blasco, J. (2001). J. Synchrotron Rad. 8, 390-392.
Sawai, H., Kokubun, J. \& Ishida, K. (2003). Photon Fact. Act. Rep. 2002, 20B, 122.

Siddons, D. P., Hart, M., Amemiya, Y. \& Hastings, J. B. (1990). Phys. Rev. Lett. 64, 1967-1970.

Sirotin, Yu. I. \& Shaskolskaya, M. P. (1975). Osnovy Kristallofiziki. Moskow: Nauka. (In Russian.)

Sirotine, Yu. \& Chaskolskaia, M. P. (1982). Fundamentals of Crystal Physics. Moscow: Mir.

Sirotine Y. \& Chaskolskaia, M. (1984). Fondements de la Physique des Cristaux. Moscow: Mir. (In French.)

Soejima, Y., Isshiki, K., Bindi, L., Ovchinnikova, E. N. \& Dmitrienko, V. E. (2003). Photon Fact. Act. Rep. 2002, 20B, 204.

Subías, G., García, J., Blasco, J., Proietti, M. G., Renevier, H. \& Sánchez, M. C. (2004). Phys. Rev. Lett. 93, 156408-1-156408-4.

Subías, G., García, J., Proietti, M. G., Blasco, J., Renevier, H., Hodeau, J. L. \& Sánchez, M. C. (2004). Phys. Rev. B, 70, 155105-1-155105-8.

Taillefumier, M., Cabaret, D., Flank, A.-M. \& Mauri, F. (2002). Phys. Rev. B, 66, 195107-1-195107-8.

Takahashi, M., Igarashi, J. \& Fulde, P. (1999). J. Phys. Soc. Jpn, 68, 2530-2533.

Templeton, D. H. (1994). Resonant Anomalous X-ray Scattering, edited by G. Materlik, C. J. Spark \& K. Fisher, pp. 1-7. Amsterdam: North-Holland.

Templeton, D. H. (1998). Acta Cryst. A54, 158-162.

Templeton, D. H. \& Templeton, L. K. (1980). Acta Cryst. A36, 237-241.

Templeton, D. H. \& Templeton, L. K. (1982). Acta Cryst. A38, $62-67$.

Templeton, D. H. \& Templeton, L. K. (1985a). Acta Cryst. A41, 133-142.

Templeton, D. H. \& Templeton, L. K. (1985b). Acta Cryst. A41, 365-371.

Templeton, D. H. \& Templeton, L. K. (1986). Acta Cryst. A42, $478-481$.

Templeton, D. H. \& Templeton, L. K. (1987). Acta Cryst. A43, 573-574.

Templeton, D. H. \& Templeton, L. K. (1989a). Acta Cryst. A45, 39-42.

Templeton, D. H. \& Templeton, L. K. (1989b). Mater. Res. Soc. Symp. Proc. 143, 177-184.

Templeton, D. H. \& Templeton, L. K. (1991). Acta Cryst. A47, 414-420.

Templeton, D. H. \& Templeton, L. K. (1992). Acta Cryst. A48, 746-751.

Templeton, D. H. \& Templeton, L. K. (1994). Phys. Rev. B, 49, 14850-14853.

Templeton, D. H. \& Templeton, L. K. (1997). Acta Cryst. A53, 352-355.

Templeton, L. K. \& Templeton, D. H. (1988). Acta Cryst. A44, 1045-1051.

Templeton, L. K. \& Templeton, D. H. (1995). J. Synchrotron Rad. 2 , 31-35.

Toda, T., Nogami, T., Yamaaki, K. \& Soejima, Y. (1998). J. Appl. Cryst. 31, 423-429.

Tsuji, E., Kurasawa, T., Yazawa, I., Katoh, H., Momozawa, N., Ishida, K. \& Kishimoto, S. (1996). J. Phys. Soc. Jpn, 65, 610-614.

Vedrinskii, R. V., Kraizman, V. L., Novakovich, A. A. \& Machavariani, V. Sh. (1992). J. Phys. Condens. Matter, 4, 6155-6169.

Watanabe, A., Ninomiya, Y., Kokubun, J. \& Ishida, K. (2001). Photon Fact. Act. Rep. 2000, 18B, 90.

Weckert, E. \& Hümmer, K. (1997). Acta Cryst. A53, 108-143.

Wilkins, S. B., Spencer, P. D., Hatton, P. D., Collins, S. P., Roper, M. D., Prabhakaran, D. \& Boothroyd, A.T. (2003). Phys. Rev. Lett. 91, 167205-1-167205-4.

Wilkinson, A. P., Templeton, L. K. \& Templeton, D. H. (1995). J. Solid State Chem. 118, 383-388. 